Article

\title{
Consumer Evaluation of Processing Variants of Pomegranate Juice
}

\section{Erika Anderson, Kadri Koppel * and Edgar Chambers IV}

The Sensory Analysis Center, Kansas State University, 1310 Research Park Drive, Manhattan, KS 66502, USA; E-Mails: elanderson@gmail.com (E.A.); eciv@ksu.edu (E.C.)

* Author to whom correspondence should be addressed; E-Mail: kadri@ksu.edu; Tel.: +1-785-532-0163; Fax: +1-785-532-3132.

Academic Editor: Jeehyun Lee

Received: 10 September 2014 / Accepted: 20 October 2014 / Published: 3 November 2014

\begin{abstract}
Increasing evidence of their health benefits has boosted the popularity of pomegranates. The effects of processing (e.g., pasteurization, drying) on pomegranate juice characteristics (e.g., color, phenolic content) and sensory attributes have been studied by several authors. The objectives of this study were to (1) understand if processing, such as pasteurization or drying, has an effect on pomegranate juice acceptance, and (2) if acceptance is related to healthy eating habits or variety seeking tendencies. Arils were removed from fresh Wonderful pomegranates for juicing or drying. Four treatments were prepared: fresh, fresh frozen, pasteurized, and reconstituted juice from dried arils. Fresh frozen, pasteurized, and reconstituted juices were evaluated by consumers for acceptance. Cluster analysis was conducted and four consumer clusters were found from evaluation of these juice treatments. Each juice was individually disliked by one of three clusters, demonstrating the effect of processing on acceptance. The fourth and largest cluster liked all three treatments. In addition, the consumers were asked to fill in Stage of Change and Variety Seeking scales. Liking scores were not found to be highly associated with healthy eating habits or variety seeking tendencies. This information is beneficial for the fruit processing industry, showing that processing can influence consumer acceptance.
\end{abstract}

Keywords: pomegranate juice; processing; flavor; consumer acceptance 


\section{Introduction}

Pomegranates, originally from Iran and the surrounding area, have spread to China, the United States, and Mediterranean countries such as Spain and Morocco. The increase in popularity of pomegranate products within the United States is largely due to health benefits [1]. Cardiovascular, anti-inflammatory, anticancer, and antidiabetic effects encompass the areas of successful in vitro and in vivo investigation. Although many positive results have been encountered, these studies would benefit from further clinical investigation [2-4]. Recent clinical trials of pomegranate juice for hemodialysis patients have shown improvements in systolic blood pressure, pulse pressure, and lipid profiles [5]. Type 2 diabetes patients have also benefited from pomegranate juice consumption by decreasing levels of inflammatory markers that play a role in cardiovascular disease [6]. Polyphenolic compounds including phenolic acids, flavonoids (e.g., anthocyanins), and tannins are largely responsible for these types of health benefits [7].

Many studies have reported processing, such as pasteurization, clarification, concentration, and dehydration effects on health beneficial phenolic compounds. The effect of pasteurization has been shown to increase or decrease anthocyanin content based on pomegranate cultivar and anthocyanin type $[8,9]$. Clarification, using chemical agents and filtration processes, is used to remove cloud from juices to improve color, flavor, and storage stability. Clarifying methods have displayed differences in levels of phenolic removal, for example gelatin has shown a greater level of total phenolic reduction while polyvinylypolypyrrolidone (PVPP) had a greater effect on anthocyanin reduction [10]. However, filtration processes, such as microfiltration, have shown a greater reduction in anthocyanin content than the use of chemical agents [11].

Reduction of available water through concentration or drying methods not only helps preserve this seasonal fruit, but also increases storage time and provides a more convenient form for shipping. Concentration methods, although resulting in anthocyanin loss, show better retention if microwave heating at high pressures is available [12]. Pomegranate powder production parameters, such as drying agent type and level as well as temperature, can also influence phenolic content. Huruz et al. [13] found anthocyanin and phenolic content to be best preserved using higher levels of both maltodextrin and temperatures during drying. However, Robert et al. [14] found that soy protein isolates prevent reduction of these compounds better than maltodextrin. Pomegranate arils are commonly dried in India for use in medicines and culinary preparations [15,16]. Singh and Sethi [16] found when drying pomegranate that the anthocyanin level concentrated resulting in a product with a higher level of anthocyanins by weight compared to fresh arils. Jaiswal et al. [17] further studied drying of arils and found that drying reduced the level of anthocyanins compared to the levels found in fresh arils. Anthocyanin reductions were greater during sun drying than oven drying.

The understanding of the effects of processing on bioactive compounds is fairly comprehensive; little investigation has taken place to understand the effects these methods have on flavor. Sensory studies have previously investigated descriptive flavor profiles of different pomegranate cultivars [18,19], pomegranate juice blends [20], and commercial products [21,22]. Consumer studies have been used to further understand the liking of differing profiles [19,20,23].

Different scales can be used in addition to acceptance questions to gain further understanding of consumers' behaviors and attitudes [24]. Understanding their interest in health related products could be assessed through methods such as food diaries, food recall, and food frequency questionnaires which can be 
lengthy and expensive approaches. However, three questions suggested by Soontrunnarudrungsri [25] can be used to segment consumers interested in healthy eating. The variety seeking scale (VARSEEK) may be used to assess a consumer's intrinsic desire to try different food products [26].

Understanding the effects processing imparts on flavor will provide information that is useful in ingredient selection. Additionally, gaining understanding of consumer liking will provide information to processors on the impact of processing methods on acceptability. If dissimilarities are apparent across processing methods, flavor profiles and consumer acceptance should be taken into consideration when selecting products for use. The objectives of this study were to (1) understand the effect of processing including freezing, batch pasteurization, and rehydration of ground, dried arils on consumer acceptability of pomegranate juices, and (2) understand if associations exist between consumer acceptability of these juices and interest in healthy eating and variety seeking tendencies.

\section{Experimental Section}

\subsection{Pomegranate Juice Treatments}

Fresh pomegranates of the Wonderful cultivar grown in the United States were acquired from Youngstown Grape Distributors (Reedley, CA, USA). The fruits were randomly divided into four groups, washed, and damaged fruits were discarded (Figure 1). Each fruit was cut in half and arils were removed manually by placing each half on top of a Seed-Out (Pahrump, NV, USA), a round raised platform with spokes to support pomegranate halves. Each half was gently tapped and arils falling below Seed-Out were removed for further processing. Any remaining arils connected to the rind were separated manually. Arils were rinsed to further remove any remaining carpellar membranes.

The arils were divided into four parts. Three of the four parts of the resultant pomegranate arils were used for juicing while the fourth was used for drying. Arils used to prepare juice were placed into a Hamilton Beach Health Smart Juice Extractor 67800 (Hamilton Beach/Proctor Silex Inc., South Pines, NC, USA). Approximately $4 \mathrm{~L}$ of fresh juice was produced in total over a period of 3 days for immediate analysis. Approximately $17 \mathrm{~L}$ was frozen fresh in $946 \mathrm{~mL}$ Ziploc freezer bags (SC Johnson, Racine, WI, USA) in a Standex BCF93558-0DX6 blast freezer (Standex, Salem, NH, USA) at $\left(-26 \pm 1{ }^{\circ} \mathrm{C}\right)$, for a minimum of $30 \mathrm{~min}$. Once frozen the treatment was transferred to a freezer for storage at $-18 \pm 1{ }^{\circ} \mathrm{C}$. Of the remaining juice, approximately $15 \mathrm{~L}$ was batch pasteurized using a Pastomaster $60 \mathrm{RTX}$ (Carpigiani, Bologna, Italy) at $63{ }^{\circ} \mathrm{C}$ for $30 \mathrm{~min}$ prior to freezing using the process described for the fresh frozen treatment. The remaining arils in the amount of $29 \mathrm{~kg}$, were dried in batches. Each batch was placed in single layers onto trays of an Excalibur 3926T Food Dehydrator (Excalibur Products, Sacramento, CA, USA). Each batch of arils was dried at $57^{\circ} \mathrm{C}$ for $10 \mathrm{~h}$ resulting in approximately $6 \mathrm{~kg}$ of dried arils. Dried arils were stored frozen in Ziploc freezer bags at $-18 \pm 1{ }^{\circ} \mathrm{C}$ until sample preparation. 


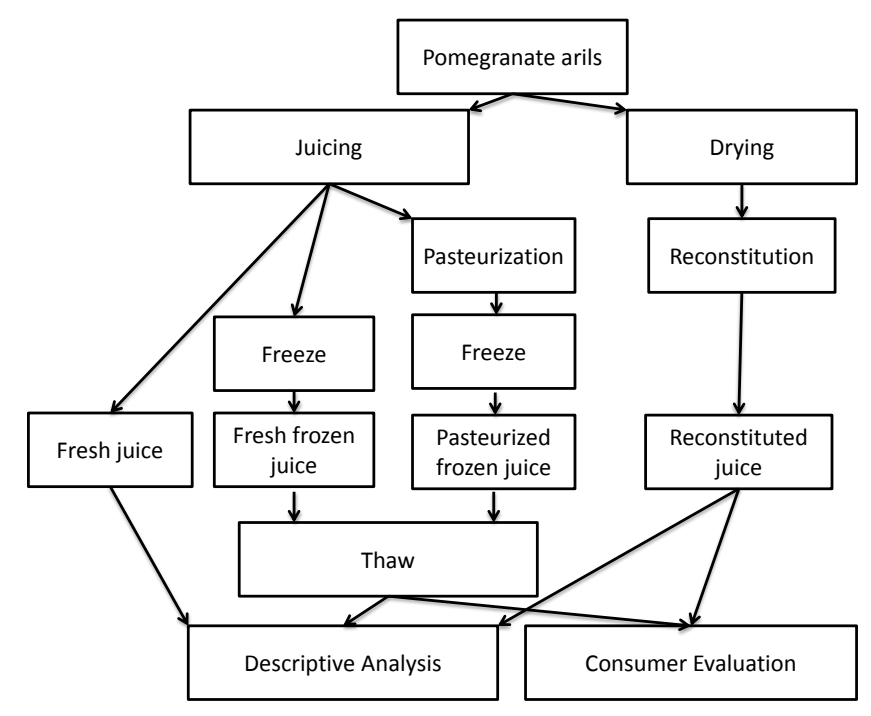

Figure 1. Pomegranate juice processing.

\subsection{Descriptive Analysis}

The four juice treatments were evaluated using a consensus modified flavor profile previously used by $[21,27,28]$. Samples were evaluated using descriptive attributes, definitions, and references modified from Koppel and Chambers [21]. A total of 27 attributes were evaluated on the ballot: apple, berry, cranberry, cherry, grape, floral, fruity, carrot, beet, brown spice, fermented, fruity-dark, green-viney, musty/earthy, brown sweet, honey, molasses, overall sweet, sweet, woody, salty, sour, bitter, metallic, astringent, toothetch, chalky mouthfeel.

Each panelist evaluated all attributes for each treatment individually on an intensity scale from 0 (none) to 15 (extremely strong). Following individual evaluation the group discussed each attribute and assigned it an intensity based on reference intensities provided. Treatments were evaluated during $1.5 \mathrm{~h}$ sessions, 2 treatment samples per session. Treatment samples were labeled using three-digit codes and served in a random order. Purified water, unsalted crackers, and low-moisture part-skim mozzarella were used as palate cleansers.

Six highly trained panelists with over $1000 \mathrm{~h}$ testing experience from the Sensory Analysis Center, Kansas State University (Manhattan, KS, USA) evaluated the treatments. The panelists had prior experience evaluating a variety of products including pomegranates and pomegranate juices. All panelists participated in orientation of the juices they were testing. Thirty milliliters of treatment was served in an odor-free, disposable $96 \mathrm{~mL}$ cup at ambient room temperature $\left(21 \pm 1{ }^{\circ} \mathrm{C}\right)$. Additional treatment samples were available upon request.

\subsection{Consumer Evaluation}

\subsubsection{Sample Preparation}

Frozen juice samples were placed in the refrigerator at $4{ }^{\circ} \mathrm{C}$ two days prior to the study to facilitate thawing. Frozen, dried arils were ground using a Black and Decker Smart Grind CBG5 (Applica Consumer Products Inc., Miami Lakes, FL, USA). The ground arils were rehydrated with purified water using a ratio of 22.2 parts arils to 78.2 parts water. The slurry of ground arils was allowed to rest for 
10 min to reach a level of 14-15 degrees brix. After resting, the solids were separated from the resulting juice by passing through a sieve two times and the brix measurement was confirmed using a MT-032 Hand Refractometer (QA Supplies Perishable and Environmental Management, Norfolk, VA, USA). A total of $30 \mathrm{~mL}$ of juice were poured into odor-free, disposable $96 \mathrm{~mL}$ cups and stored in the refrigerator at $4{ }^{\circ} \mathrm{C}$ prior to evaluation. Treatment samples were served at $5 \pm 1{ }^{\circ} \mathrm{C}$.

\subsubsection{Consumers}

Consumers were recruited from the Manhattan, Kansas area to participate in pomegranate juice evaluation. Initial screening was performed using Compusense at-hand ${ }^{\circledR}$ (Compusense Inc., Guelph, Ontario, Canada). The 100 consumers that participated met all eligibility requirements including: had no food allergies, drank juice a minimum of 2 to 3 times a week and were willing to try pomegranate juice. All consumers were scheduled by telephone and received a confirmation email of the scheduled participation time. In total, $45 \%$ males and $55 \%$ females participated in the consumer study (Table 1).

Table 1. Consumer demographics.

\begin{tabular}{cccc}
\hline Gender & Consumer \% & Age Range & Consumer \% \\
\hline Female & 55 & $18-24$ & 14 \\
Male & 45 & $25-34$ & 19 \\
& & $35-44$ & 15 \\
& & $45-54$ & 21 \\
& & $55-64$ & 29 \\
\hline
\end{tabular}

\subsubsection{Test Design and Sample Evaluation}

Compusense at-hand ${ }^{\circledR}$ (Compusense Inc., Guelph, Ontario, Canada) was used to generate the test design that was balanced for carryover effects. All positions and orders were blocked by groups of six for a possible 120 consumers.

Evaluation of treatments was completed in a total of six sessions over a two-day period; each consumer participated in one of the six sessions. Before beginning the session, consumers were provided with information regarding the study and the procedures that would be used during the testing session. The consumers then electronically signed consent forms stating that they agreed to participate in the study and could withdraw at any time without penalty. Each session lasted for approximately $30 \mathrm{~min}$. Consumers were provided with purified water and unsalted crackers for use in between treatment samples, and optional cups for expectoration.

Compusense at-hand ${ }^{\circledR}$ (Compusense Inc., Guelph, Ontario, Canada) was used on iPads to collect data. Panelists first confirmed their demographic information. Then treatment samples were presented monadically according to each panelists serving order. The consumers rated each sample using five categories of liking including: overall, fruit flavor, sweetness, sourness, and aftertaste. A nine-point hedonic scale ranging from dislike extremely to like extremely was used to indicate level of liking.

After completing sample evaluation, consumers rated their association with the eight statements on the VARSEEK scale using a five point scale; ranging from completely disagree (1) to completely agree (5) [26]. The consumers were grouped based on their scores into three groups: a low (score $\leq 25$ ), medium 
(26-34), and high ( $\geq 35$ ). The scale consisted of the following statements: (1) When I eat out, I like to try the most unusual items, even if I am not sure I would like them, (2) While preparing foods or snacks, I like to try out new recipes, (3) I think it is fun to try out food items one is not familiar with, (4) I am eager to know what kinds of foods people from other countries eat, (5) I like to eat exotic foods, (6) Items on the menu that I am unfamiliar with make me curious, (7) I prefer to eat food products that I am used to, and 8) I am curious about food products I am not familiar with.

Additionally, each consumer answered three questions described by Soontrunnarudrungsri [25] regarding their habits towards a healthy diet: (1) Do you think you usually eat a healthy diet overall? (options included: yes/no), (2) Do you plan to change your diet to be healthier? (options included: Yes, I am considering changing my overall diet in the next month to be more healthful; Yes, I am considering changing my overall diet in the next 6 months to be more healthful; No, I have no plans to change my overall diet to increase its healthfulness), and (3) How long ago did you change and start eating a healthier diet overall? (options included: I made serious changes in my overall diet to be more healthful in the past 6 months; I made changes to a more healthful diet and have eaten that way for 6 months or more). The first question asks the consumer if they regularly consume a healthy diet. Upon answering "no" the consumer is then asked if they plan to change their diet to be healthier. If not planning to change their diet they were considered to be in the Precontemplation stage. If planning to change in the next month to 6 months they were considered to be in the Preparation and Contemplation stages respectively. If answering "yes" to the first question and making serious changes in the last 6 months they were considered in the Action stage. If indicating a healthy diet for longer than 6 months they were considered in the Maintenance stage [25].

After the session was completed, consumers were monetarily compensated for their time to participate in the study.

\subsubsection{Statistical Analysis}

Analysis of Variance (ANOVA) was used to test if significant differences existed at a 5\% significance level between juice treatment liking scores. Ward's minimum variance procedure was performed to determine if distinct groups existed among consumers. ANOVA with post-hoc means separation using Fisher's protected LSD was used to evaluate significant differences at the 5\% significance level across all treatments' liking scores by variety seeking level, across all juice liking scores by healthy eating interest, and across all juice liking scores and variety seeking scores for all clusters. All analyses were performed using SAS ${ }^{\circledR}$ statistical software (version 9.3, SAS Institute Inc., Cary, NC, USA).

\section{Results and Discussion}

\subsection{Results}

\subsubsection{Descriptive Evaluation}

The descriptive analysis results were described by Koppel et al. [29]. Briefly, it was found that pomegranate juice samples did not differ as much in their magnitude of each attribute as they did in the number of attributes (Table 2). The fresh juice did not display many fluctuations in comparison to the 
fresh frozen juice. The most noticeable difference in fresh juice was the lack of carrot flavor, apparent in the other treatments. Although musty/earthy and molasses flavors did not occur in the fresh frozen juice, these flavors were present in the pasteurized and reconstituted juice. The pasteurized juice, in comparison to the fresh frozen, lacked apple flavor, was lower in cherry flavor and astringency, and higher in grape, dark fruity, and brown sweet flavors intensities. The reconstituted juice was the most differentiated showing slight characteristics of brown spice and fermented with lack of beet, green-viney, and honey flavors.

Table 2. Modified consensus flavor profile scores for pomegranate juice samples.

\begin{tabular}{|c|c|c|c|c|}
\hline \multirow[t]{2}{*}{ Attribute } & \multicolumn{4}{|c|}{ Samples } \\
\hline & $\mathrm{F}$ & $\mathrm{FF}$ & $P$ & $\mathrm{R}$ \\
\hline Apple & $1.5 *$ & 2 & 0 & 1.5 \\
\hline Berry & 3 & 2.5 & 3 & 3 \\
\hline Cranberry & 3 & 3.5 & 3.5 & 3 \\
\hline Cherry & 2 & 3 & 1.5 & 2 \\
\hline Grape & 2 & 2 & 3 & 2 \\
\hline Floral & 3 & 2.5 & 3 & 3 \\
\hline Fruity & 4 & 4 & 4 & 3.5 \\
\hline Carrot & 0 & 1.5 & 2 & 2 \\
\hline Beet & 2 & 2 & 2.5 & 0 \\
\hline Brown Spice & 0 & 0 & 0 & 1.5 \\
\hline Fermented & 0 & 0 & 0 & 1.5 \\
\hline Fruity-Dark & 2.5 & 2 & 3 & 3.5 \\
\hline Green-Viney & 1.5 & 2 & 1.5 & 0 \\
\hline Musty/Earthy & 1.5 & 0 & 2 & 1.5 \\
\hline Brown Sweet & 2 & 2 & 3 & 3 \\
\hline Honey & 1.5 & 2 & 1.5 & 0 \\
\hline Molasses & 1.5 & 0 & 2 & 2 \\
\hline Sweet, Overall & 4 & 4 & 4 & 4.5 \\
\hline Sweet & 2.5 & 2.5 & 3 & 3 \\
\hline Woody & 2 & 2.5 & 2 & 2.5 \\
\hline Salty & 1.5 & 2.5 & 1.5 & 2.5 \\
\hline Sour & 3 & 3.5 & 3.5 & 3.5 \\
\hline Bitter & 3.5 & 3.5 & 3.5 & 3.5 \\
\hline Metallic & 2 & 2 & 2.5 & 1.5 \\
\hline Astringent & 4 & 5 & 4 & 4 \\
\hline Toothetch & 2.5 & 2.5 & 2 & 2 \\
\hline Chalky Mouthfeel & 2 & 2 & 2 & 2.5 \\
\hline
\end{tabular}

$\mathrm{F}=$ Fresh, $\mathrm{FF}=$ Fresh Frozen, $\mathrm{P}=$ Pasteurized, $\mathrm{R}=$ Reconstituted. $*$ Scores given on a scale from 0 to 15 with 0.5 increments.

\subsubsection{Consumer Acceptance and Clusters}

According to the average liking scores for each category assessed, no significant differences were present at a 5\% significance level (Figure 2). Further assessment of liking scores was performed to determine if liking clusters existed. Total of four clusters were discovered (Figure 3). Consumer 
comments overall reflected their cluster preference and scores in the different liking categories. The consumers in Cluster 3 did not display a distinct preference for any of the samples. Cluster 1 particularly disliked the fresh frozen juice, Cluster 2 disliked the rehydrated juice, and Cluster 4 disliked the pasteurized juice.

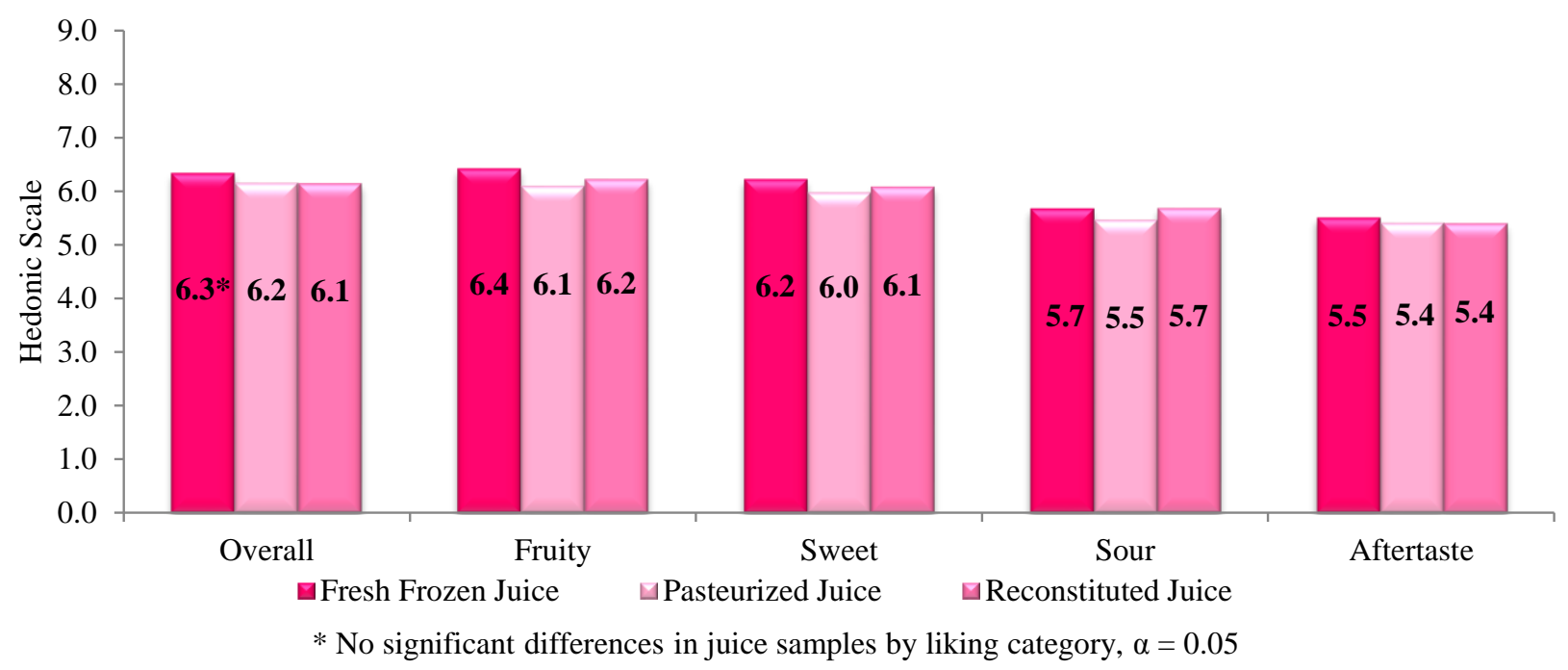

Figure 2. Average consumer liking scores of pomegranate juices. Evaluation on a 9-point hedonic scale, 1 -dislike extremely, 9-like extremely.

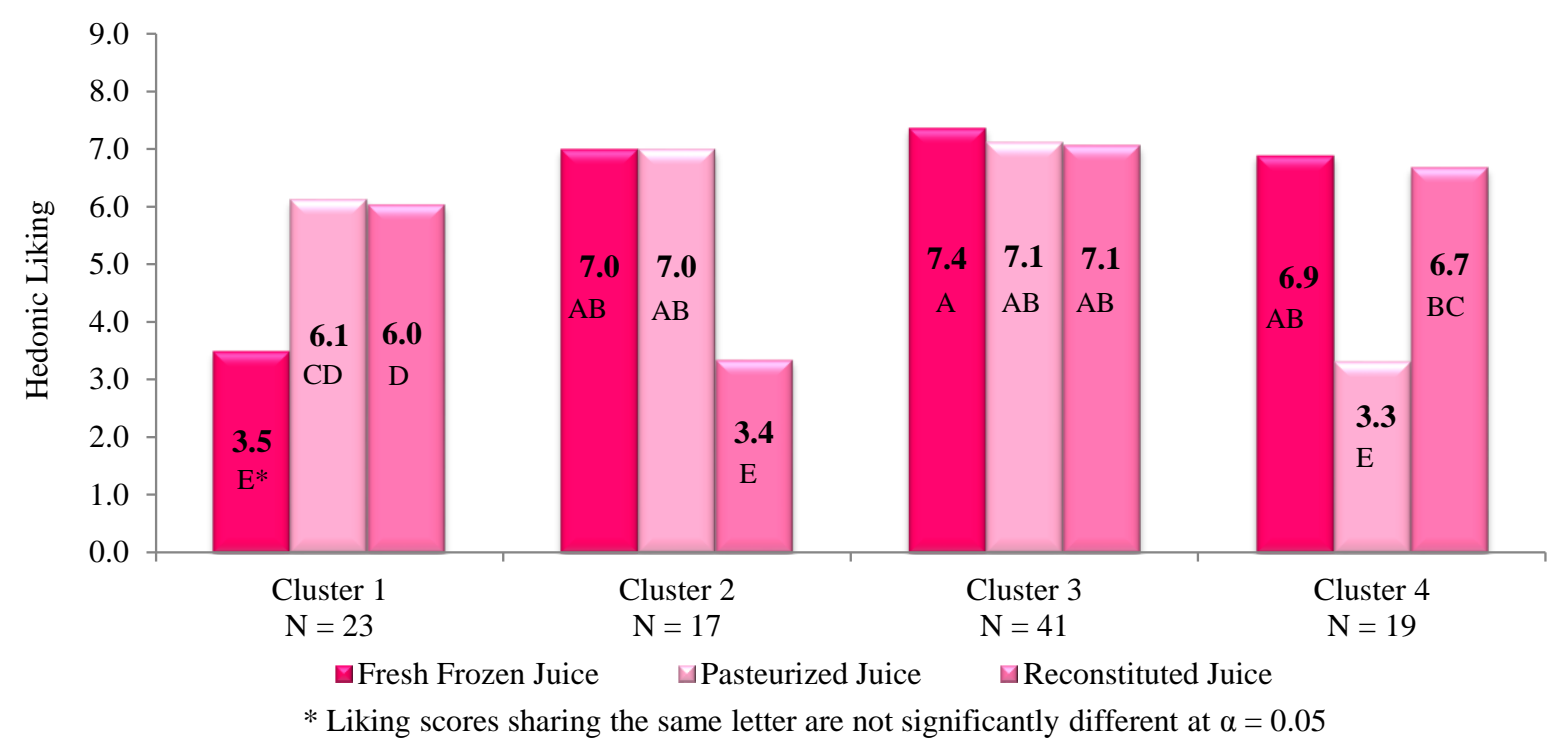

Figure 3. Average overall liking of pomegranate juices by consumer cluster. Evaluation on a 9-point hedonic scale, 1-dislike extremely, 9-like extremely.

The majority of the consumers $(80 \%)$ felt that they usually eat a healthy diet. The consumers' interest in healthy eating results can be explained by the Stages of Change model [25]. The distribution of consumers throughout the Stages of Change model reflected the results previously found by Soontrunnarudrungsri [25] showing that most of the consumers fall in the Maintenance stage, decreasing in percentage to the Contemplation stage (Table 3). However, no participants in this study were found to be in the Precontemplation stage where they felt their diet was not usually healthy and were not 
considering changing to a more healthful diet. Overall liking scores showed that the consumers in the Action stage disliked the pasteurized and reconstituted juices significantly less than the consumers in the Contemplation stage liked the pasteurized juice (Figure 4).

Table 3. Consumer interest in healthy eating habits according to the stages of change questionnaire [25].

\begin{tabular}{|c|c|c|}
\hline Stage of Change & Description & $\%$ Consumers \\
\hline Maintenance & Healthy diet for 6 months or more & 61 \\
\hline Action & $\begin{array}{l}\text { Made changes in last } 6 \text { months to be } \\
\text { more healthful }\end{array}$ & 19 \\
\hline Preparation & $\begin{array}{l}\text { Considering to a more healthful diet } \\
\text { in next month }\end{array}$ & 13 \\
\hline Contemplation & $\begin{array}{l}\text { Considering changing to a more } \\
\text { healthful diet in the next } 6 \text { months }\end{array}$ & 7 \\
\hline
\end{tabular}

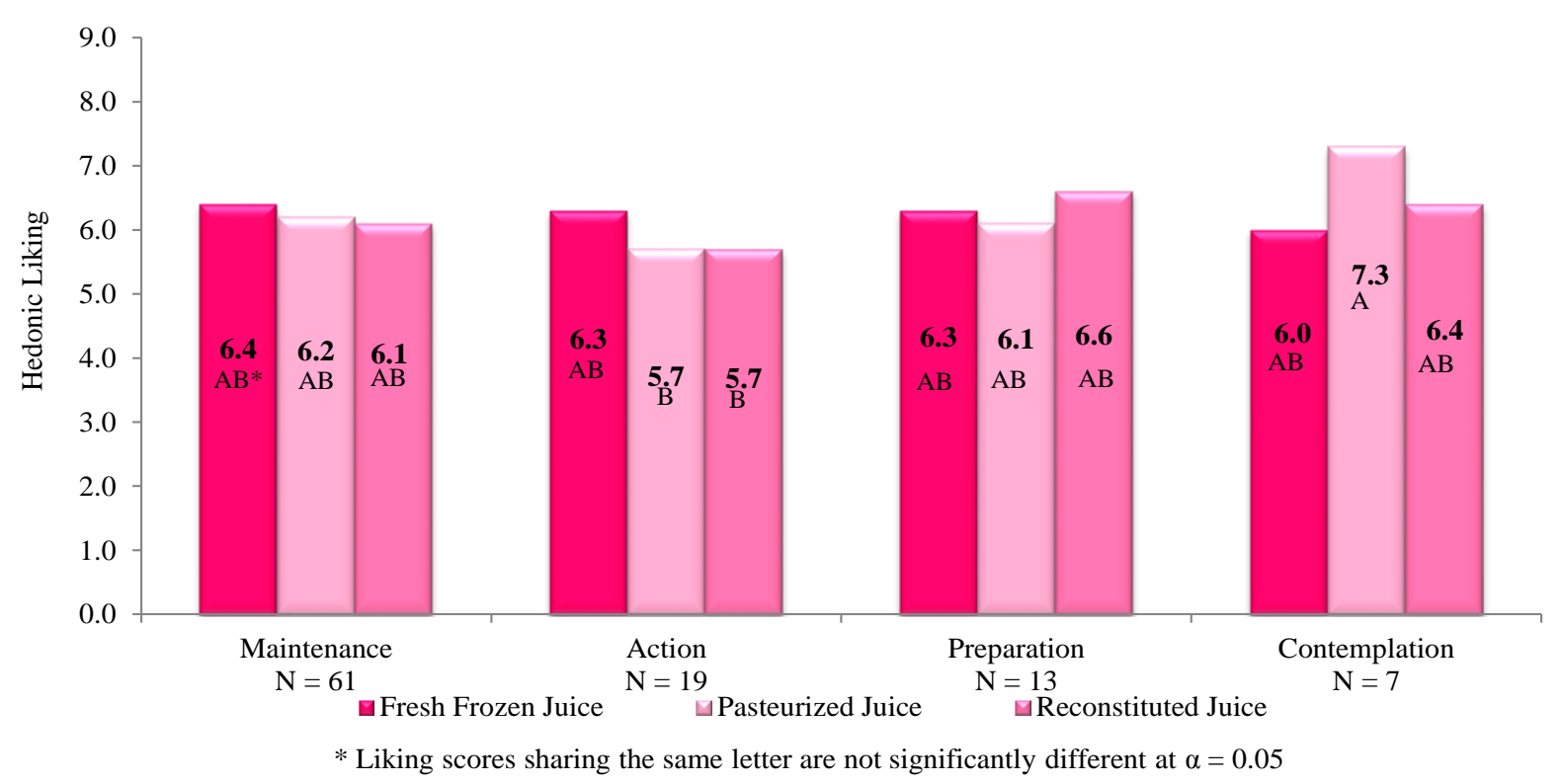

Figure 4. Average overall liking scores by stages of change scale [25]. Evaluation on a 9-point hedonic scale, 1 - dislike extremely, 9-like extremely.

Van Trijp and Steenkamp [26] have divided the possible variety seeking scores into the levels of low, medium, and high (Table 4). The average consumer in this study was considered to have a medium intrinsic variety seeking level with a score of 30.33. Based on these category divisions, liking scores were compared across low, medium, and high variety seeking groups. None of the variety seeking groups showed a strong level of liking for any of the juice types (Figure 5). However, compared to medium variety seekers, low variety seekers liked the fresh frozen juice significantly less. 
Table 4. Consumer variety seeking scores. scores calculated according to VARSEEK scale [26]. Scale 1-disagree completely to 5-agree completely, maximum value of 40.

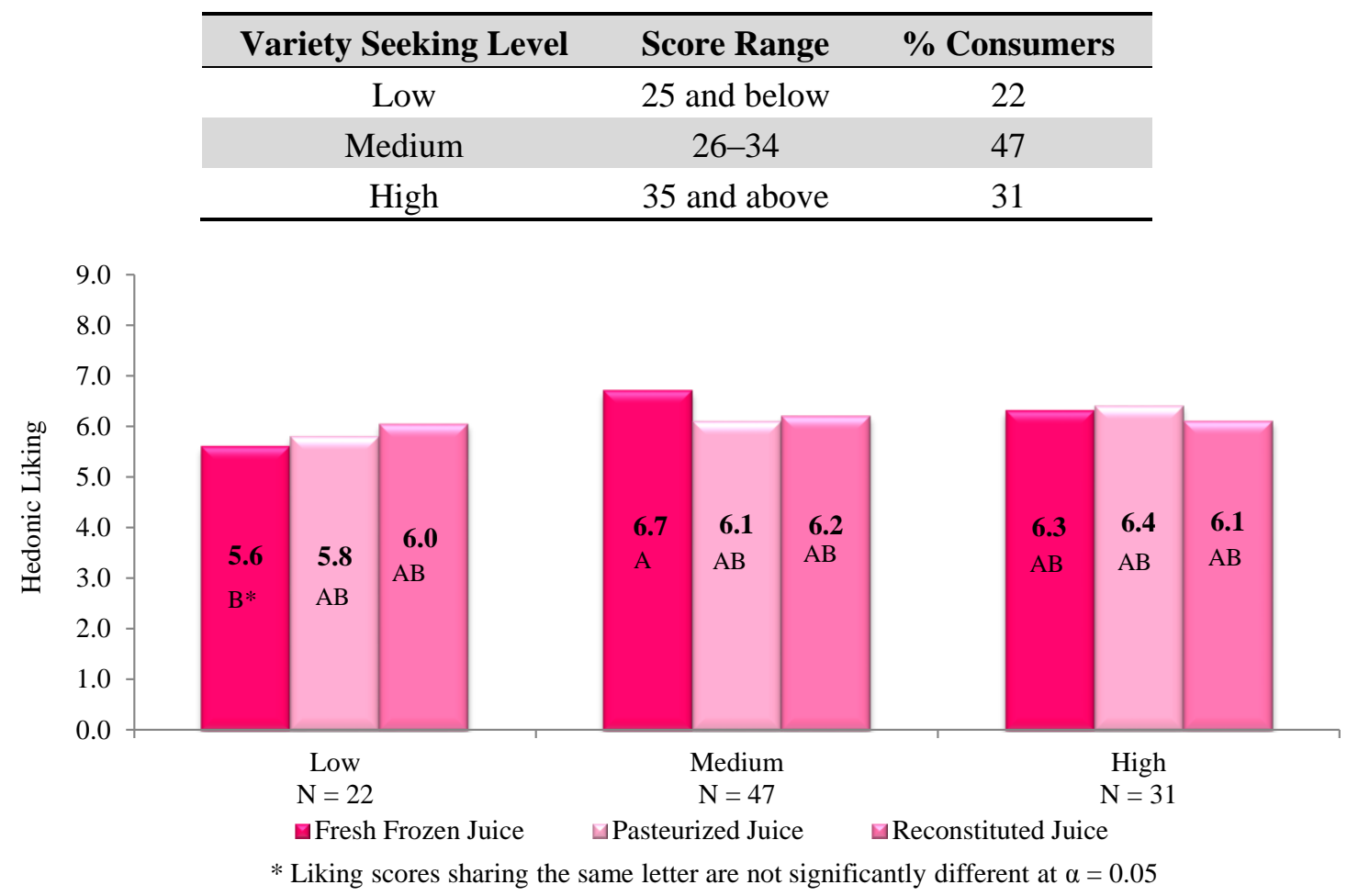

Figure 5. Average overall liking of pomegranate juices by variety seeking category. Evaluation on a 9-point hedonic scale, 1-dislike extremely, 9-like extremely.

\subsection{Discussion}

The profiles of the fresh and fresh frozen juices were found to be quite similar which allows for comparison of pasteurized and reconstituted juice to the fresh frozen juice as similar to fresh during the consumer study. Although differences were not large in magnitude, differences did exist among samples for each processing treatment. These differences probably had an influence on consumer acceptability.

Consumer clusters were expected as Koppel et al. [23] previously found different liking clusters across countries. The juices in this study were surprisingly well liked, particularly by Cluster 3 , as previous studies had not seen as high of liking scores in pomegranate juices or juice blends [23,30,31], and a consumer cluster was expected that did not like any samples as found by Koppel et al. [23]. Our finding that groups individually each disliked a treatment indicated that processing methods may play a role in consumer choice when pursuing pomegranate products. From the treatments evaluated, the appearance of specific flavors such as brown spice and fermented and lack of beet, green-viney, and honey may have contributed to the significant dislike of the reconstituted product by Cluster 2. A summation of small changes may have influenced consumer significant dislike of the pasteurized treatment by Cluster 4 . These small changes included lack of apple flavor, lower cherry flavor and salty taste, increased grape and musty/earthy flavor.

Processing exhibited changes that may be associated with heating such as increase in level of grape, dark fruity, or brown sweet flavors. Flavors such as these were previously noted in commercial juices that had undergone processing [22]. The elevation of these flavors may have influenced the liking of 
Cluster 1, which significantly disliked the fresh frozen juice in comparison to those that had undergone processing. Lozano [32] suggested that pasteurization at higher temperatures (such as that greater than $121{ }^{\circ} \mathrm{C}$ for $0.1 \mathrm{~s}$ ) may impart cooked flavor to juice. Further processing may have the capability of enhancing specific notes while decreasing others potentially impacting consumer acceptability. Future studies may address this potential by examining variables such as pasteurization temperature as it relates to product liking.

Stages of change related to interest in healthy eating showed significant differences in acceptance of pomegranate juices in the action and contemplation stages. Examining scores in the other liking categories these differences appear to relate to aftertaste. The pasteurized juice received the highest score in aftertaste liking from the Contemplation group at 6.7. While the pasteurized and reconstituted juices received the lowest aftertaste score from the Action group at 4.5 and 4.8 respectively. These groups showed a trend in aftertaste liking; the Action group was the least accepting of aftertaste, the Maintenance group was the second least accepting, and preparation and Contemplation groups were the most accepting. From this study, we observed that the majority of juice drinkers tend to be more interested in healthy eating and did not discriminate much among juices. Future studies may consider investigating if consumers who are more interested in healthful eating are more discriminatory among other foods and beverages.

VARSEEK scores were expected to be higher with higher liking of samples, as pomegranate juice popularity is relatively recent. We found that acceptability was not well distinguished by VARSEEK scores as there were no significant differences among clusters in this respect and few differences among liking of the treatments at different variety seeking levels. Previously, Lähteenmäki and van Trijp [33] also were unable to relate variety seeking tendencies to liking in the evaluation of sandwiches, but did find that those with higher variety seeking tendencies tried more "less appropriate" sandwich fillings than those with lower scores. Based on the previous results with sandwiches, it may be useful to consider evaluation of liking scores in combination with juice purchasing habits for a variety of juices to better understand the variety seeking tendencies of pomegranate juice consumers. Moreover, consumer demographics did not demonstrate large differences across clusters, including interest in healthy eating. All clusters had consumers that typically ate a healthy diet overall and if they did not believe they had a healthy diet overall were planning to change their diet to be more healthful.

These findings present additional opportunities for further understanding of sensory effects of pomegranate processing on consumer acceptance. The treatments used in this study were prepared on a small scale. As differences were present in both pasteurization and drying effects, comparison of products produced on a larger scale would be beneficial to understand processing influences in industry. Additionally, processes such as clarification and concentration should be considered for testing as noted changes are produced by polyphenol reduction and heating processes respectively. Both processes are used in industry to improve storage stability. Clarification reduces phenolic compounds decreasing the level of astringency [11]. Methods that involve heating increase the browning index, an indicator of browning reactions such as in Maillard browning or caramelization, forming new flavor compounds [12]. Astringency level fluctuation and flavor changes due to browning may impact consumer acceptance. Lawless et al. [20] found that presentation of antioxidant information influenced acceptance of black cherry, Concord grape, and pomegranate juice blends. Several studies have proven that processing has 
an influence on polyphenols; these influences could be presented to consumers as antioxidant levels to determine their impact on acceptance.

\section{Conclusions}

Flavor variations were found as a result of processing methods although small in magnitude. The amount of attributes presenting small changes is influential, presenting distinct differences in consumer liking. Most juice drinkers tend to be interested in healthy eating; however, they did not discriminate much among juices overall. Future investigations may consider examining if this is the case with other product types. Variety seeking tendencies did not show a defined relationship with product liking scores. However, the variation in processing methods exposed three consumer clusters that each individually disliked a particular treatment within the study. The largest cluster surprisingly liked all three juices at a level not previously seen in other studies. Further exploration in the areas of pomegranate processing on consumer acceptance such as full scale processing changes, additional processing methods, and antioxidant information influence would be beneficial to explore.

\section{Author Contributions}

E.A., K.K., and E.C. planned and designed the experiments; E.A. performed the experiments; E.A., K.K., and E.C. analyzed the data; E.A. wrote the manuscript, K.K. and E.C. edited the manuscript.

\section{Conflicts of Interest}

The authors declare no conflict of interest.

\section{References}

1. Hummer, K.E.; Pomper, K.W.; Postman, J.; Graham, C.J.; Stover, E.; Mercure, E.W.; Aradhya, M.; Crisosto, C.H.; Ferguson, L.; Thompson, M.M.; et al. Chapter 4: Emerging fruit crops. In Fruit Breeding; Badenes, M.L., Byrne, D.H., Eds.; Springer: New York, USA, 2012; Volume 8, pp. 97-147.

2. Faria, A.; Calhau, C. The bioactivity of pomegranate: Impact on health and disease. Crit. Rev. Food Sci. Nutr. 2011, 51, 626-634.

3. Johanningsmeier, S.D.; Harris, G.K. Pomegranate as a functional food and nutraceutical source. Annu. Rev. Food Sci. Technol. 2011, 2, 181-201.

4. Viuda-Martos, M.; Fernández-López, J.; Pérez-Álvarez, J.A. Pomegranate and its many functional components as related to human health: A review. Compr. Rev. Food Sci. Food Saf. 2010, 9, 635-654.

5. Shema-Didi, L.; Kristal, B.; Sela, S.; Geron, R.; Ore, L. Does Pomegranate intake attenuate cardiovascular risk factors in hemodialysis patients? Nutr. J. 2014, 13, 1-8.

6. Sohrab, G.; Nasrollahzadeh, J.; Zand, H.; Amiri, Z.; Tohidi, M.; Kimiagar, M. Effects of pomegranate juice consumption on inflammatory markers in patients with type 2 diabetes: A randomized, placebo-controlled trial. J. Res. Med. Sci. 2014, 19, 215-220.

7. Tzulker, R.; Glazer, I.; Bar-Ilan, I.; Holland, D.; Aviram, M.; Amir, R. Antioxidant activity, polyphenol content, and related compounds in different fruit juices and homogenates prepared from 29 different pomegranate accessions. J. Agric. Food Chem. 2007, 55, 9559-9570. 
8. Turfan, Ö.; Türkyılmaz, M.; Yemiş, O.; Özkan, M. Anthocyanin and color changes during processing of pomegranate (Punica granatum L., cv. Hicaznar) juice from sacs and whole fruit. Food Chem. 2011, 129, 1644-1651.

9. Alighourchi, H.; Barzegar, M.; Abbasi, S. Anthocyanins characterization of 15 Iranian pomegranate (Punica granatum L.) varieties and their variation after cold storage and pasteurization. Eur. Food Res. Technol. 2008, 227, 881-887.

10. Vardin, H.; Fenercioğlu, H. Study on the development of pomegranate juice processing technology: Clarification of pomegranate juice. Nahrung 2003, 47, 300-303.

11. Fischer, U.A.; Dettmann, J.S.; Carle, R.; Kammerer, D.R. Impact of processing and storage on the phenolic profiles and contents of pomegranate (Punica granatum L.) juices. Eur. Food Res. Technol. 2011, 233, 797-816.

12. Yousefi, S.; Emam-Djomeh, Z.; Mousavi, S.M.A.; Askari, G.R. Comparing the effects of microwave and conventional heating methods on the evaporation rate and quality attributes of pomegranate (Punica granatum L.) juice concentrate. Food Bioprocess Technol. 2012, 5, 1328-1339.

13. Horuz, E.; Altan, A.; Maskan, M. Spray drying and process optimization of unclarified pomegranate (Punica granatum) juice. Drying Technol. 2012, 30, 787-798.

14. Robert, P.; Gorena, T.; Romero, N.; Sepulveda, E.; Chavez, J.; Saenz, C. Encapsulation of polyphenols and anthocyanins from pomegranate (Punica granatum) by spray drying. Int. J. Food Sci. Technol. 2010, 45, 1386-1394.

15. Kingsly, A.R.P.; Singh, D.B.; Manikantan, M.R.; Jain, R.K. Moisture dependent physical properties of dried pomegranate seeds (Anardana). J. Food Eng. 2006, 75, 492-496.

16. Singh, D.; Sethi, V. Screening of pomegranate genotypes for the preparation of quality grade anardana. J. Food Sci. Technol. 2003, 40, 236-238.

17. Jaiswal, V.; DerMarderosian, A.; Porter, J.R. Anthocyanins and polyphenol oxidase from dried arils of pomegranate (Punica granatum L.). Food Chem. 2010, 118, 11-16.

18. Andreu-Sevilla, A.J.; Mena, P.; Martí, N.; García-Viguera, C.; Carbonell-Barrachina, Á.A. Volatile composition and descriptive sensory analysis of pomegranate juice and wine. Food Res. Int. 2013, $54,246-254$.

19. Carbonell-Barrachina, A.A.; Calín-Sánchez, A.; Bagatar, B.; Hernández, F.; Legua, P.; Martínez-Font, R.; Melgarejo, P. Potential of Spanish sour-sweet pomegranates (cultivar C25) for the juice industry. Food Sci. Technol. Int. 2012, 18, 129-138.

20. Lawless, L.J.R.; Threlfall, R.T.; Meullenet, J.; Howard, L.R. Applying a mixture design for consumer optimization of black cherry, concord grape and pomegranate juice blends. J. Sens. Stud. 2013, 28, 102-112.

21. Koppel, K.; Chambers IV, E. Development and application of a lexicon to describe the flavor of pomegranate juice. J. Sens. Stud. 2010, 25, 819-837.

22. Vázquez-Araújo, L.; Koppel, K.; Chambers IV, E.; Adhikari, K.; Carbonell-Barrachina, A.A. Instrumental and sensory aroma profile of pomegranate juices from the USA: Differences between fresh and commercial juice. Flavour Fragrance J. 2011, 26, 129-138.

23. Koppel, K.; Chambers IV, E.; Vázquez-Araújo, L.; Timberg, L.; Carbonell-Barrachina, Á.; Suwonsichon, S. Cross-country comparison of pomegranate juice acceptance in Estonia, Spain, Thailand, and United States. Food. Qual. Prefer. 2014, 31, 116-123. 
24. Lawless, H.T.; Heymann, H. Chapter 14: Acceptance Testing: 14.6 Behavioral and Context-Related Approaches. In Sensory Evaluation of Food Principles and Practices; Springer: New York, NY, USA, 2010; pp. 340-343.

25. Soontrunnarudrungsri, A. Development and Validation of Screening Tools for Classification Consumers of Food Products Based on Eating Healthy Criteria. Ph.D. Thesis, Kansas State University, Manhattan, KS, USA, December, 2011.

26. Van Trijp, H.C.; Steenkamp, J.E. Consumers' variety seeking tendency with respect to foods: Measurement and managerial implications. Eur. Rev. Agric. Econ.1992, 19, 181-195.

27. Cherdchu, P.; Chambers IV, E.; Suwonsichon, T. Sensory lexicon development using trained panelists in Thailand and the USA: Soy sauce. J. Sens. Stud. 2013, 28, 248-255.

28. Di Donfrancesco, B.; Koppel, K.; Chambers IV, E. An initial lexicon for sensory properties of dry dog food. J. Sens. Stud. 2012, 27, 498-510.

29. Koppel, K.; Anderson, E.L.; Chambers IV, E. Influence of processing on pomegranate (Punica granatum L.) juice flavor and aroma. J. Sci. Food Agric. 2014, in press.

30. Vázquez-Araújo, L.; Chambers IV, E.; Adhikari, K.; Carbonell-Barrachina, Á.A. Sensory and physicochemical characterization of juices made with pomegranate and blueberries, blackberries, or raspberries. J. Food Sci. 2010, 75, S398-S404.

31. Endrizzi, I.; Pirretti, G.; Calò, D.G.; Gasperi, F. A consumer study of fresh juices containing berry fruits. J. Sci. Food Agric. 2009, 89, 1227-1235.

32. Lozano, J.E. Processing of fruits: Elevated temperature, nonthermal, and miscellaneous processing. In Fruit Manufacturing: Scientific Basis, Engineering Properties, and Deteriorative Reactions of Technological Importance; Springer: New York, USA, 2006; pp. 55-71.

33. Lähteenmäki, L.; van Trijp, H. Hedonic responses, variety-seeking tendency and expressed variety in sandwich choices. Appetite 1995, 24, 139-152.

(C) 2014 by the authors; licensee MDPI, Basel, Switzerland. This article is an open access article distributed under the terms and conditions of the Creative Commons Attribution license (http://creativecommons.org/licenses/by/4.0/). 